\title{
Nanoparticle-targeted Drug
}

National Cancer Institute

\section{Source}

National Cancer Institute. Nanoparticle-targeted Drug. NCI Thesaurus. Code C48203.

A complex consisting of a therapeutic entity coupled to a nanoparticle entity that has an external coating designed to deliver the therapeutic to a precise location in the body. This complex is useful because of the ability to deliver drugs in a controlled fashion to a specific site of interest. Conjugates such as this are also utilized as imaging and contrast agents for MRI and other imaging studies. 